\title{
THE FORMATION OF IRIS DIAGNOSTICS. I. A QUINTESSENTIAL MODEL ATOM OF Mg II AND GENERAL FORMATION PROPERTIES OF THE Mg II h\&k LINES
}

\author{
J. Leenaarts ${ }^{1}$, T. M. D. Pereira ${ }^{1,2,3}$, M. Carlsson ${ }^{1}$, H. Uitenbroek ${ }^{4}$, And B. De Pontieu ${ }^{1,3}$ \\ ${ }^{1}$ Institute of Theoretical Astrophysics, University of Oslo, P.O. Box 1029, Blindern, N-0315 Oslo, Norway; \\ jorrit1@astro.uio.no, tiago.pereira@astro.uio.no,mats.carlsson@astro.uio.no,bdp@1msal.com \\ 2 NASA Ames Research Center, Moffett Field, CA 94035, USA \\ ${ }^{3}$ Lockheed Martin Solar \& Astrophysics Laboratory, Org. A021S, Building 252, 3251 Hanover Street, Palo Alto, CA 94304, USA \\ ${ }^{4}$ NSO/Sacramento Peak, P.O. Box 62, Sunspot, NM 88349-0062, USA; huitenbroek@nso.edu \\ Received 2013 April 4; accepted 2013 June 3; published 2013 July 10
}

\begin{abstract}
NASA's Interface Region Imaging Spectrograph (IRIS) space mission will study how the solar atmosphere is energized. IRIS contains an imaging spectrograph that covers the Mg II h\&k lines as well as a slit-jaw imager centered at $\mathrm{Mg}$ II $\mathrm{k}$. Understanding the observations will require forward modeling of $\mathrm{Mg}$ II h\&k line formation from three-dimensional (3D) radiation-MHD models. This paper is the first in a series where we undertake this forward modeling. We discuss the atomic physics pertinent to h\&k line formation, present a quintessential model atom that can be used in radiative transfer computations, and discuss the effect of partial redistribution (PRD) and $3 \mathrm{D}$ radiative transfer on the emergent line profiles. We conclude that $\mathrm{Mg}$ II h\&k can be modeled accurately with a four-level plus continuum $\mathrm{Mg}$ II model atom. Ideally radiative transfer computations should be done in 3D including PRD effects. In practice this is currently not possible. A reasonable compromise is to use one-dimensional PRD computations to model the line profile up to and including the central emission peaks, and use 3D transfer assuming complete redistribution to model the central depression.
\end{abstract}

Key words: radiative transfer - Sun: atmosphere - Sun: chromosphere

Online-only material: color figures

\section{INTRODUCTION}

Magnesium is one of the most abundant elements in the solar atmosphere and as such its neutral and singly ionized state gives rise to a number of spectral lines with significant diagnostic potential in the photosphere as well as in the chromosphere. Well-known examples are the Mg I $457.1 \mathrm{~nm}$ forbidden line, which is a photospheric temperature diagnostic, the $\operatorname{Mg} \mathrm{I} b$ lines around $517 \mathrm{~nm}$, which serve as upper photospheric/lower chromospheric magnetic field diagnostics, the Mg I $12 \mu \mathrm{m}$ infrared lines, which exhibit very peculiar non-LTE radiative transfer behavior (Chang et al. 1991; Carlsson et al. 1992), the $285.2 \mathrm{~nm} \mathrm{Mg}$ I resonance line, which shows effects of partial frequency redistribution (PRD; Uitenbroek \& Briand 1995), and finally the $\mathrm{Mg}$ II $\mathrm{h} \& \mathrm{k}$ resonance doublet at 280.27 and $279.55 \mathrm{~nm}$, respectively. The latter two lines are among the strongest, and therefore potentially most valuable diagnostically, in the solar spectrum, but have rarely been observed because of their wavelength in the middle of the UV range, precluding ground-based observation.

This situation will change drastically with the NASA's Interface Region Imaging Spectrograph (IRIS) spacecraft, which will be equipped with a high-resolution UV imaging spectrograph ( $<80 \mathrm{~m} \AA$ spectral resolution) and an $\mathrm{Mg}$ II $\mathrm{k}$ slit-jaw imager (4 Å filter width) with a 0.'4 spatial resolution.

Previously, disk center calibrated intensities have been obtained with the Hawaii (Allen \& McAllister 1978) and Harvard (Kohl \& Parkinson 1976) sounding rocket experiments. Low temporal and spatial resolution (2"), but high spectral resolution $(20 \mathrm{~m} \AA$ ) spectra have been obtained with the Ultraviolet Spectrometer and Polarimeter (Woodgate et al. 1980) instrument on board the Solar Maximum Mission (SMM; Vial 1984). The lines have been observed simultaneously with other chromospheric spectral lines in the UV ( $\operatorname{Ly} \alpha$ and $\operatorname{Ly} \beta$, and the Ca II $\mathrm{H} \& \mathrm{~K}$ lines) by the LPSP instrument on board the $O S O 8$ mission
(Bonnet et al. 1978; Artzner et al. 1978; Vial et al. 1979, 1981; Kneer et al. 1981). Spatially resolved spectra of the Mg I $285.2 \mathrm{~nm}$ (Uitenbroek \& Briand 1995) and Mg II h\&k lines were obtained with the French balloon experiment RASOLBA (Staath \& Lemaire 1995) and the sounding rocket experiment HRTS on its ninth flight (Morrill \& Korendyke 2008), both on a photographic film. Polarimetric observations in the $\mathrm{h} \& \mathrm{k}$ lines were obtained with two flights of the Solar Ultraviolet Magnetograph Investigation (West et al. 2011) sounding rocket experiment.

Because magnesium is about 18 times more abundant than calcium (Asplund et al. 2009), the h\&k lines form correspondingly higher in the solar atmosphere than the homologous Ca II H\&K lines. From the sparse observations described above, it is clear that the magnesium resonance lines sample a different regime of the solar atmosphere than the $\mathrm{H} \& \mathrm{~K}$ lines, which regularly lack emission reversals altogether, or exhibit singly peaked profiles (Rezaei et al. 2008), whereas the former lines always have doubly peaked emission reversals, except in sunspots (Morrill et al. 2001). In addition, the strong red-blue asymmetry of the Ca II H\&K lines that is thought to be the result of acoustic waves steepening into shocks (Carlsson \& Stein 1997) is much less pronounced in the h\&k lines (Gouttebroze 1989). Since the magnetic network gives rise to more symmetric and less intermittent $\mathrm{Ca}$ II $\mathrm{H} \& \mathrm{~K}$ line profiles, the question arises if the magnetic field also gives rise to the omnipresent emission reversals in the h\&k lines, or whether the different morphology of the emission in the latter lines can be explained by the same dynamic effects that cause the intermittent $\mathrm{Ca}$ II behavior, given that the magnesium lines sample a different height due to their larger opacity.

Slight differences in atomic structure between singly ionized calcium and magnesium create an interesting opportunity for simultaneous temperature and velocity diagnostics in the spectral window sampled by the IRIS spectrograph. Whereas the 
Ca II $3 d^{2} D$ levels have a lower energy by about $1.3 \mathrm{eV}$ than the $4 p^{2} P$ upper levels of the H\&K lines, and thus allow for the $4 d^{2} D-4 p^{2} P$ triplet to form in the infrared, the $\mathrm{Mg}$ II $3 d^{2} D$ levels lie at about the same energy difference above the $3 p^{2} P$ levels as these levels lie above the $\mathrm{Mg}$ II $3 s^{2} S$ ground state. As a result, the $3 d^{2} D-3 p^{2} P$ triplet forms in the UV, very close to the h\&k resonance lines, overlapping with them in wavelength.

Because of their high opacity (magnesium is almost exclusively in its singly ionized state as shown in Section 6.2), radiation in the $\mathrm{Mg}$ II resonance and $3 d^{2} D-3 p^{2} P$ triplet emanates from the low-density chromosphere, where non-LTE radiative transfer and effects of PRD are crucial to line formation. Previously, the Mg II lines had been modeled with PRD in onedimensional (1D) fashion and compared with observations by Milkey \& Mihalas (1974), Uitenbroek (1997), and Gouttebroze (1989), among others. Inversion of the spectra that the IRIS mission will provide into physical quantities is therefore a complicated endeavor and is best achieved by comparing forward modeling of spectra through numerical simulations of radiation magnetohydrodynamics (RMHD) with observations, and using such simulations to extract what observable quantities mean in terms of physical quantities of the underlying atmosphere. In this paper, we therefore present a new updated study of the line formation properties of the $\mathrm{Mg}$ II $\mathrm{h} \& \mathrm{k}$ lines incorporating the latest advances in numerical modeling of the solar chromosphere and non-LTE radiative transfer to help interpretation of IRIS data. To do so we use the same snapshot of an RMHD simulation as Leenaarts et al. (2012a), who studied the formation of the $\mathrm{H} \alpha$ line. The reader is encouraged to compare their results with the results for $\mathrm{Mg}$ II h\&k in the current paper.

In Section 2, we describe the atomic data that we use to construct our Mg II model atom. Section 3 describes the model atmospheres we use, Section 4 describes the employed radiative transfer codes. In Section 5 we investigate the importance of non-equilibrium ionization of magnesium, in Section 6 we describe the construction of our quintessential model atom, i.e., the smallest model atom that accurately reproduces the $\mathrm{h} \& \mathrm{k}$ line, in Sections 7-11 we describe the basic h\&k line formation properties in some detail, and we finish with our discussion and conclusions in Section 12.

\section{ATOMIC DATA}

We use the magnesium abundance of $A_{\mathrm{Mg}}=7.60 \pm 0.04$ on the standard logarithmic scale for abundances where $A_{\mathrm{H}}=$ 12 from Asplund et al. (2009). These authors remark that there is no agreement between abundance determinations from different lines, and the stated abundance is a straight mean of the abundance determinations for many lines computed of Mg I and Mg II in non-LTE using one-dimensional (1D) model atmospheres (Zhao et al. 1998; Abia \& Mashonkina 2004).

The atomic energy levels are taken from the NIST database (Ralchenko et al. 2011).

The oscillator strengths and photoionization cross sections are taken from the TOPbase database ${ }^{5}$ (Cunto et al. 1993). Note that some of the photoionization cross sections provided by TOPbase extend below the ionization threshold energy. These data points are added for the purpose of average opacity computations, but should be discarded for non-LTE radiative transfer computations as in this paper (C. Mendoza 2012, private communication).

Excitation by collisions with electrons is treated with data from Sigut \& Pradhan (1995). Collisional excitation and de-

\footnotetext{
5 http://cdsweb.u-strasbg.fr/topbase/topbase.html
}

excitation between the h\&k upper levels by collisions with neutral hydrogen are included according to Monteiro et al. (1988). These authors give tabulated values up to $5000 \mathrm{~K}$, we use a linear extrapolation above this temperature.

Autoionization, collisional ionization from the ground state by electrons, and charge exchange with protons and neutral hydrogen are done using the recipes by Arnaud \& Rothenflug (1985).

Dielectronic recombination is treated according to Shull \& van Steenberg (1982) and collisional ionization from excited states is computed using the procedure of Burgess \& Chidichimo (1983).

Broadening of the $\mathrm{Mg}$ II h\&k lines by collisions with neutral hydrogen is treated with the formalism of Anstee \& O'Mara (1995) using the pertinent coefficients from Barklem \& O'Mara (1998).

When we discuss the effect of inclusion of $\mathrm{Mg}$ I in the atomic model on the emergent intensity from the Mg II h\&k lines, we use a model atom including $\mathrm{Mg}$ I, $\mathrm{Mg}$ II, and the ground state of $\mathrm{Mg}$ III. This atom is created by adding the $\mathrm{Mg}$ I model atom of Carlsson et al. (1992) to our Mg II model.

\section{MODEL ATMOSPHERES}

In this paper, we use a number of different model atmospheres. One is the semi-empirical model $\mathrm{C}$ from Fontenla et al. (1993). This is a 1D time-independent atmosphere, constructed to match spatially and temporally averaged observed spectra in the UV. This atmosphere is used as a starting point in understanding h\&k line formation in a simple, static case. This understanding can then be used in the analysis of more complicated model atmospheres. We henceforth refer to this model as FALC.

The second model is a snapshot of a three-dimensional (3D) RMHD simulation performed with the Bifrost code (Gudiksen et al. 2011). The same snapshot has been used by Leenaarts et al. (2012a) to investigate $\mathrm{H} \alpha$ line formation.

Bifrost solves the equations of resistive MHD on a staggered Cartesian grid including a variety of physical processes. The simulation we use here included optically thick radiative transfer in the photosphere and low chromosphere (Nordlund 1982; Skartlien 2000; Hayek et al. 2010), parameterized radiative losses in the upper chromosphere, transition region and corona (Carlsson \& Leenaarts 2012), thermal conduction along magnetic field lines (Gudiksen et al. 2011), and an equation of state that includes the effects of non-equilibrium ionization of hydrogen (Leenaarts et al. 2007).

The simulation covers a physical extent of $24 \times 24 \times 16.8 \mathrm{Mm}$, with a grid of $504 \times 504 \times 496$ cells, covering the upper convection zone, photosphere, chromosphere, and the lower corona. The horizontal grid spacing is $48 \mathrm{~km}$, the vertical grid spacing is non-uniform, with a spacing of $19 \mathrm{~km}$ between $z=-1$ and $z=5 \mathrm{Mm}$, and increasing toward the bottom and top of the computational domain. The simulation contains a magnetic field with an average unsigned strength of $50 \mathrm{G}$ in the photosphere, concentrated in the photosphere in two clusters of opposite polarity. We shall refer to the snapshot from this simulation as 3DMHD.

We also use a snapshot from a two-dimensional (2D) simulation performed with Bifrost by Leenaarts et al. (2011). This simulation was performed with the same physics as 3DMHD, but had a very weak magnetic field $(0.3 \mathrm{G}$ average unsigned flux in the photosphere). It has a grid size of $325 \times 512$ points, with a horizontal grid spacing of $32.5 \mathrm{~km}$ spanning $16.6 \mathrm{Mm}$ 
and a non-uniform vertical grid with a spacing of $28 \mathrm{~km}$ between the bottom of the computational domain and $z=5 \mathrm{Mm}$, and increasing toward the top of the computational domain to $150 \mathrm{~km}$.

Characteristic of this $2 \mathrm{D}$ simulation is that it contains large bubbles of low-temperature $(<2000 \mathrm{~K})$ gas in the chromosphere, which do not occur in 3DMHD. At these low temperatures, we expect a significant amount of magnesium in the form of $\mathrm{Mg}$, so we use the $2 \mathrm{D}$ simulation to extend the range of physical circumstances under which we study $\mathrm{Mg}$ II h\&k line formation. We call this snapshot 2DMHD.

\section{RADIATIVE TRANSFER}

We perform non-LTE radiative transfer computations with two different codes. The first is a modified version of $R H$ by Uitenbroek (2001). This version was parallelized using MPI so that it can efficiently solve the radiative transfer in all columns in a 3D model atmosphere assuming each column is a plane-parallel 1D atmosphere. It can treat the effects of angle-dependent partial frequency redistribution (PRD) using the fast approximation by Leenaarts et al. (2012b). We employ a non-constant coherency fraction $\gamma$, given by

$$
\gamma=\frac{P_{j}}{P_{j}+Q_{j}^{\mathrm{E}}},
$$

where $P_{j}$ is the total (collisional and radiative) rate out of the upper level of the transition $j$ and $Q_{j}^{\mathrm{E}}$ is the rate of elastic collisions for the upper level. For further details on the treatment of PRD, we refer to Uitenbroek (2001).

The second code we use is Multi3d (Leenaarts \& Carlsson 2009). This is an MPI-parallelized radiative transfer code that can evaluate the radiation field in full $3 \mathrm{D}$, taking the horizontal structure in the model atmosphere into account. The current iterative solution methods for non-LTE radiative transfer with PRD effects are not stable enough to include in 3D. Our computations with Multi3d are done assuming complete redistribution (CRD). This assumption gives wrong line intensities from the wings up to and including the line core emission peaks $\left(\mathrm{k}_{2}\right.$ and $\left.\mathrm{h}_{2}\right)$. However, we show in Section 10 that the central line depressions $\left(\mathrm{k}_{3}\right.$ and $\left.\mathrm{h}_{3}\right)$ are only weakly affected by PRD.

For the FALC model, we add additional spectral line broadening using the height-dependent microturbulent velocity given in Fontenla et al. (1991). Computations using the 3DMHD and 2DMHD atmospheres do not use microturbulence.

Summarizing, we model the h\&k lines in 1D including PRD with the $R H$ code, and in 3D assuming CRD with Multi3d. Ideally, one should perform 3D computations including PRD, but this is currently beyond our capabilities.

\section{NON-EQUILIBRIUM IONIZATION}

Hydrogen ionization is out of equilibrium in the dynamic solar chromosphere due to long ionization/recombination timescales compared with dynamical timescales (Carlsson \& Stein 2002; Leenaarts et al. 2007). If the same is true for magnesium, it would be necessary to take into account the history of the atmosphere when interpreting $\mathrm{Mg}$ II spectra.

We here investigate the timescales of ionization/ recombination of $\mathrm{Mg}$ II-Mg III using the same methodology as in Carlsson \& Stein (2002). We use a time series of 1D hydrodynamical snapshots computed with RADYN (e.g., Carlsson \&
Stein 1992, 1997). This code self-consistently solves the equations of conservation of mass, momentum, energy, and charge together with the non-LTE rate equations for hydrogen, helium, and calcium. The equations are formulated on an adaptive grid (Dorfi \& Drury 1987) that ensures high numerical resolution where the gradients are large (e.g., in shock fronts). We use the same time series as in Carlsson \& Stein (2002) and refer the reader to that paper for details on the numerical implementation.

For each snapshot in the time series, we perturb the atmosphere by increasing the temperature by $1 \%$ and calculate the time evolution of the population densities. We use a 10 level plus continuum model atom for $\mathrm{Mg}$ II. Its term diagram is given in Figure 1. We determine the timescale for ionization/recombination by fitting the time evolution of the Mg III population density with the analytic solution for a two-level atom:

$$
n(t)=n(\infty)+(n(0)-n(\infty)) \mathrm{e}^{-t / t_{\text {relax }}},
$$

where $n(t)$ is the population density at time $t, n(\infty)$ is the equilibrium population density of the perturbed atmosphere, $n(0)$ is the population density of the initial atmosphere, and $t_{\text {relax }}$ is the timescale for relaxation to the final state.

Figure 2 shows the relaxation time and the temperature as a function of column mass. The relaxation time increases from milliseconds in the photosphere to about $50 \mathrm{~s}$ at the top of the chromosphere. Note, however, that this dynamic simulation starts from a radiative equilibrium atmosphere and it thus has a low temperature in the chromosphere apart from when shocks travel through. In the shocks, the relaxation time is lowered by one order of magnitude compared with the pre-shock, lowtemperature state (e.g., at $t=1760 \mathrm{~s}$ when there is a shock at $\left.\lg \left(m_{c}\right)=-3.7\right)$. In the transition region, the relaxation timescale drops to fractions of a second. We conclude that whenever the temperature is high enough to give a significant fraction of $\mathrm{Mg}$ III, the relaxation time is short and statistical equilibrium is a good approximation for magnesium.

\section{QUINTESSENTIAL MODEL ATOM}

In this section, we investigate the effect of the size of the model atom on the emergent $\mathrm{Mg}$ II h\&k line profiles.

\subsection{Starting Atom}

As a starting atomic model for Mg II, we use the same 10 level plus continuum model atom as in Section 5. Its term diagram is given in Figure 1. The h\&k lines at $279.55 \mathrm{~nm}$ and $280.27 \mathrm{~nm}$ are lines from the $3 s^{2} S$ ground state to the $3 p^{2} P$ excited states. There is a triplet of lines between the $3 p^{2} P$ and $3 d^{2} D$ states with wavelengths close to the h\&k lines. One has a wavelength of $279.08 \mathrm{~nm}$ and is located on the blue side of the k core, the other two are overlapping lines at $279.79 \mathrm{~nm}$ and $279.80 \mathrm{~nm}$ and are located in between the $\mathrm{h} \& \mathrm{k}$ line cores. This triplet can potentially be used as an upper-photospheric/low-chromospheric velocity indicator. We plan to investigate the diagnostic value of the triplet in a future paper. The $4 \mathrm{~d}, 4 \mathrm{p}$, and $4 \mathrm{f}$ terms are merged into single levels. Their energy is computed as an average of the energy of the levels in the term, weighted with their statistical weight.

\subsection{Importance of $\mathrm{Mg} I$}

A fraction of all magnesium atoms in the solar atmosphere is in the form of $\mathrm{Mg}$ I. The ionization balance is sensitive to the 


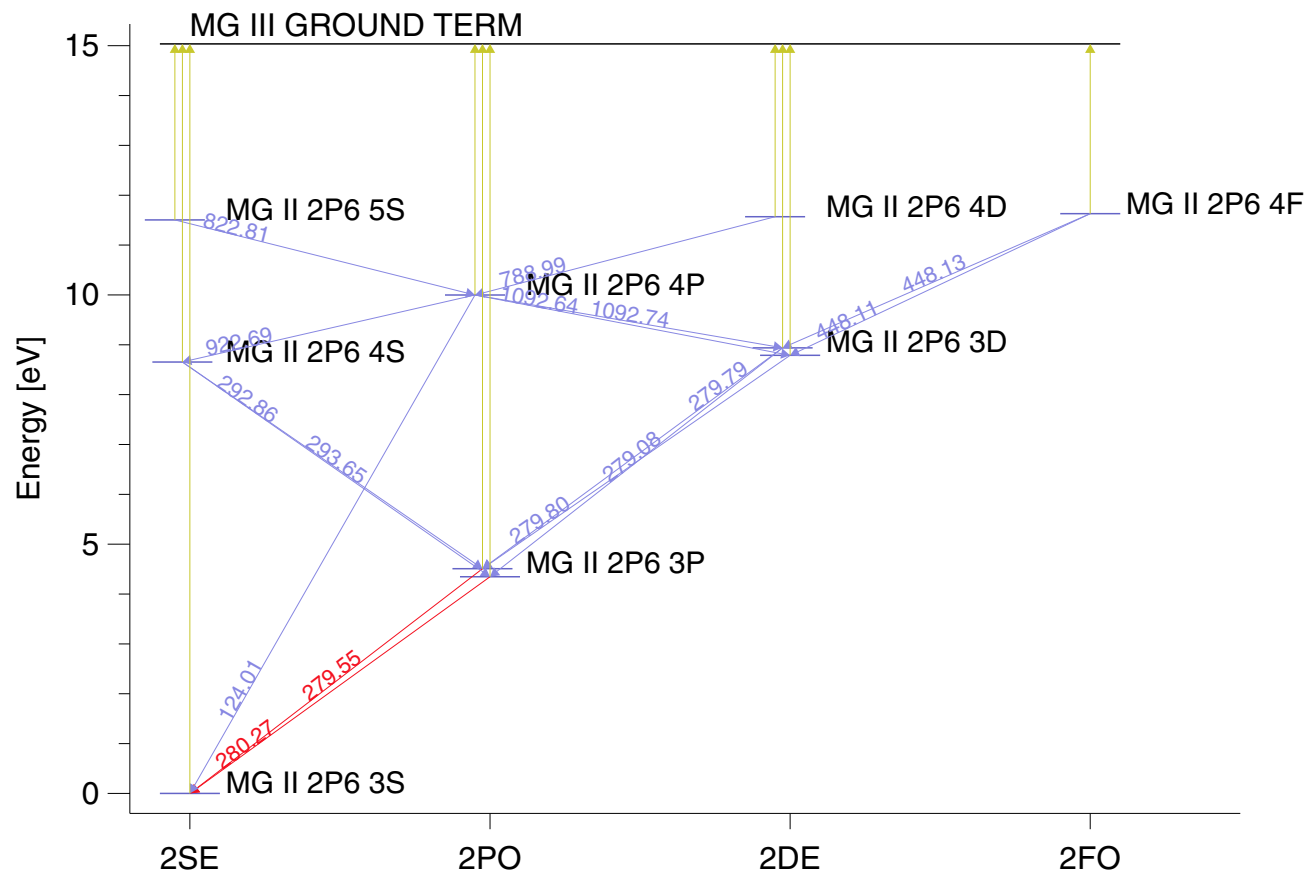

Figure 1. Term diagram of the 10 level plus continuum $\mathrm{Mg}$ II atomic model. The $\mathrm{Mg}$ II h\&k lines are indicated by red lines, all other bound-bound transitions are blue, and the bound-free transitions are yellow. All lines indicating bound-bound transitions are annotated with the line-center wavelength in nanometers.

(A color version of this figure is available in the online journal.)

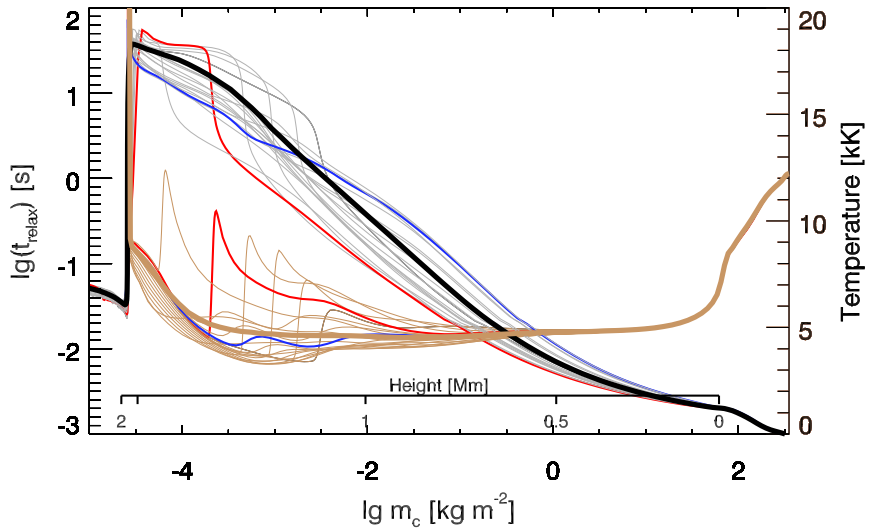

Figure 2. Relaxation timescale (gray/black, left axis) and gas temperature (brown, right axis) as function of column mass and time in the dynamic simulation at $10 \mathrm{~s}$ intervals from $t=1600 \mathrm{~s}$ to $t=1780 \mathrm{~s}$. The thickest lines represent the relaxation timescale and the temperature averaged in time over all time steps between $t=1120 \mathrm{~s}$ and $t=3590 \mathrm{~s}$. Two individual time steps are drawn as thick lines: $t=1640 \mathrm{~s}$ (red) and $t=1760 \mathrm{~s}$ (blue). The height scale of the initial state is given as reference.

(A color version of this figure is available in the online journal.)

temperature and electron density, through the Saha-equation in LTE and the ionization and recombination rate coefficients in non-LTE (cf. Mihalas 1978; Rutten 2003). It is thus expected that the fraction of magnesium in its neutral form is highest in cool gas at a high density.

The addition of $\mathrm{Mg}$ I levels to our Mg II model atom causes a large performance penalty due to the large increase in the number of levels and transitions. This penalty is so large that it would hamper computation of the h\&k lines from time series of large 3D RMHD simulations.

We therefore investigated whether $\mathrm{Mg}$ I should be included in non-LTE for accurate computation of the h\&k line profiles. We created a model atom including neutral, singly ionized and twice ionized magnesium by adding the 65 level $\mathrm{Mg}$ I atom of
Carlsson et al. (1992) to the Mg II model, including all pertinent transitions.

We then compared the non-LTE radiative transfer problem between this large model atom and our small 10 level plus continuum $\mathrm{Mg}$ II atom where we only included the 65 level $\mathrm{Mg}$ I atom as a source of background opacity assuming LTE. Including $\mathrm{Mg}$ I in LTE in the background does not significantly increase the amount of computational work.

We used $R H$ to solve the radiative transfer problem for both model atoms in three plane-parallel atmospheres: FALC, a column of 3DMHD, and a column of 2DMHD. From the latter model, we specifically chose a column with a low temperature throughout most of the chromosphere and photosphere, thus representing a case with a large influence of $\mathrm{Mg}$ I on the $\mathrm{h} \& \mathrm{k}$ profiles.

The results are shown in Figure 3. Panels (a)-(c) show the temperature structure in the models and panels (d)-(f) show the fraction of all magnesium in the various ionization states. As expected, the fraction of $\mathrm{Mg} \mathrm{I}$ is largest in cool areas in the photosphere, but only in the extremely cool 2DMHD case it exceeds $10 \%$. Above $1 \mathrm{Mm}$ height, at most $1 \%$ of magnesium is in the form of neutral atoms.

Panels (g)-(1) show the effect on the emergent line profiles. If Mg $\mathrm{I}$ is treated as an LTE background element, then the opacity in the wavelength range of the $\mathrm{h} \& \mathrm{k}$ lines is increased owing to the larger fraction of Mg I in LTE compared to non-LTE. In the $\mathrm{h} \& \mathrm{k}$ line cores, the $\mathrm{Mg}$ I opacity is insignificant. In the outer line wings, however, $\mathrm{Mg}$ I opacity is important, especially in the red wing of $\mathrm{Mg}$ II $\mathrm{h}$ because of the presence of the $\mathrm{Mg}$ I resonance line at $285.2 \mathrm{~nm}$.

Treating Mg I in LTE leads to higher wing opacity. With a source function that decreases with height this results in a lower h\&k wing intensity. The cores of the lines are, however, completely unaffected.

We therefore conclude that it is safe to ignore $\mathrm{Mg}$ I if one is interested mainly in the chromosphere where the h\&k line 
FALC
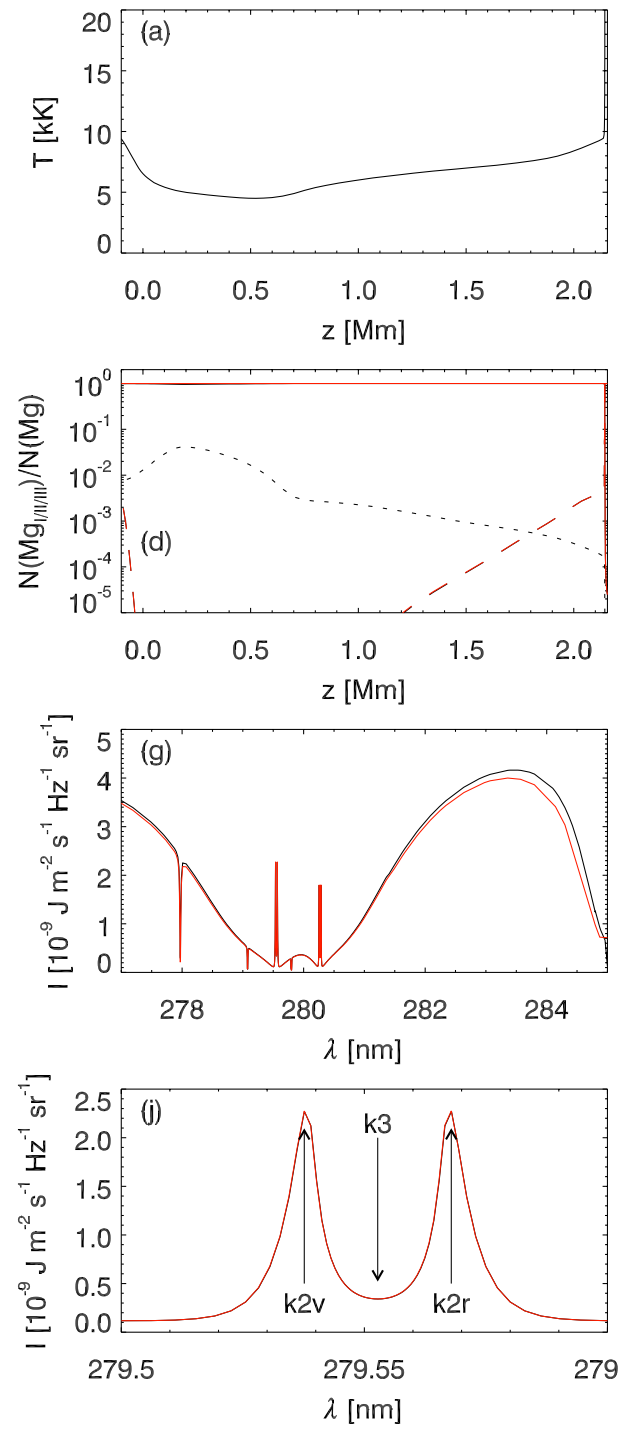

3DMHD
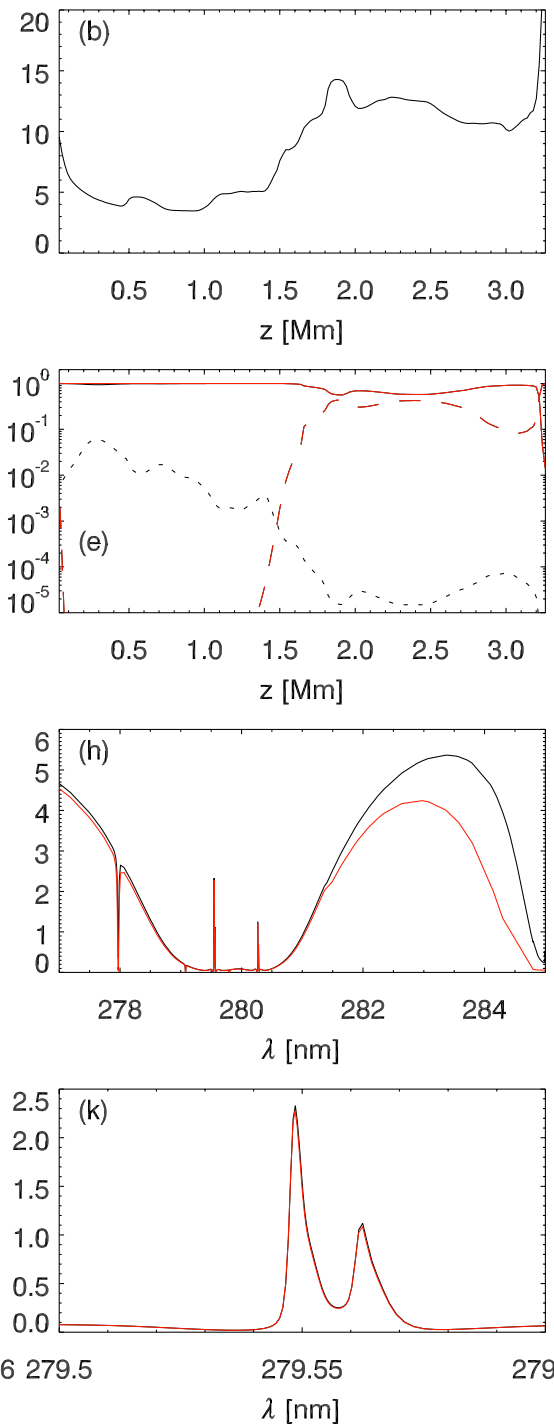

2DMHD
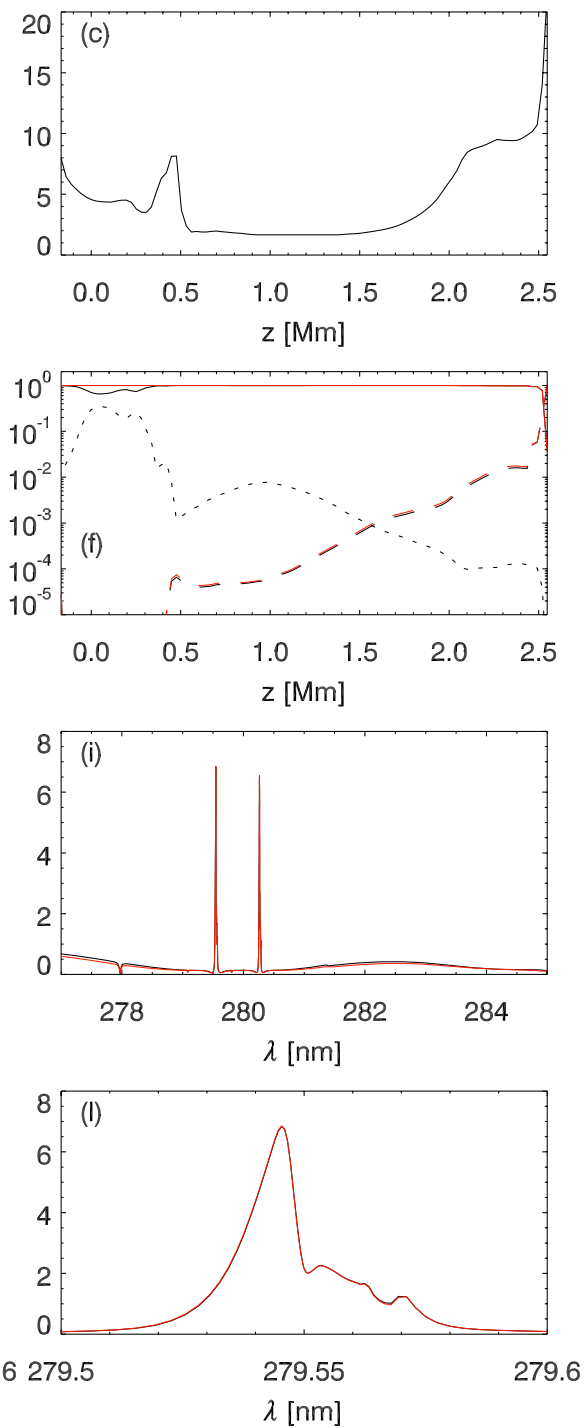

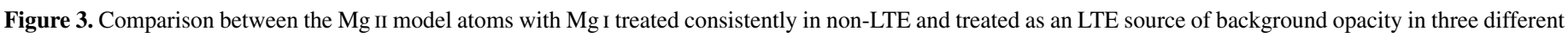

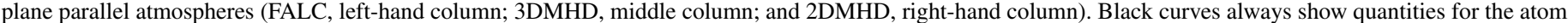

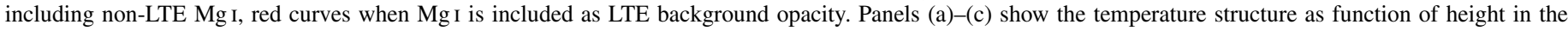

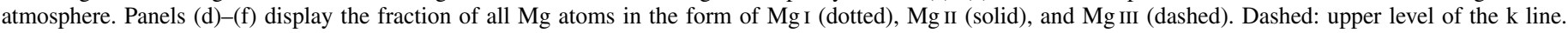

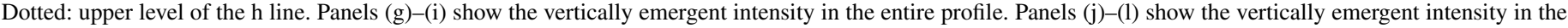

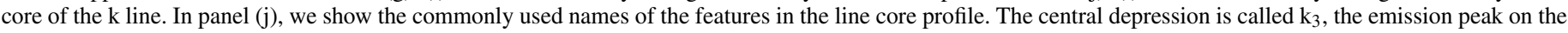
short-wavelength (blue) side of the depression is called $\mathrm{k}_{2 V}$, and the peak on the red side is called $\mathrm{k}_{2 R}$.

(A color version of this figure is available in the online journal.)

cores are formed. If one is interested in precise computation of the outer line wing intensity, then Mg I should be included in non-LTE. Note that the line wings are blended with many lines, mainly from iron, which then also should be included. We focus here mainly on the properties of h\&k line formation relevant for the IRIS mission, so we ignore $\mathrm{Mg}$ I for the remainder of this paper.

\subsection{Influence of Number of Levels in the Mg II Atom}

The 10 level plus continuum $\mathrm{Mg}$ II atom contains 15 bound-bound and 10 bound-free radiative transitions. Especially for 3D computations, it would be beneficial to construct a model atom with fewer levels and transitions to speed up computations. To do so, we investigated the various rates in our 10+1 level magnesium atom and show the results of this analysis in
Figure 4 for the FALC model (left-hand column) and a column of 3DMHD (right-hand column).

The top row shows the net rate $P_{\text {net }}$ out of the h\&k upper levels ( $3 p^{2} P$ states):

$$
P_{\text {net }}=\frac{1}{n_{\mathrm{Mg}}} \sum_{i} \sum_{j}\left(n_{i} P_{i j}-n_{j} P_{j i}\right),
$$

where the $i$ summation is over the two upper levels of h\&k and the summation over $j$ is over the target states, $n_{\mathrm{Mg}}$ is the total density of magnesium atoms, $n_{i}$ is the population of level $i$, and $P_{i j}$ is the total (collisional plus radiative) rate from level $i$ to level $j$. In the deepest part of the atmosphere, the h\&k lines are in detailed balance, but toward the top of the atmosphere the h\&k upper levels are populated from the higher-lying excited 
FALC
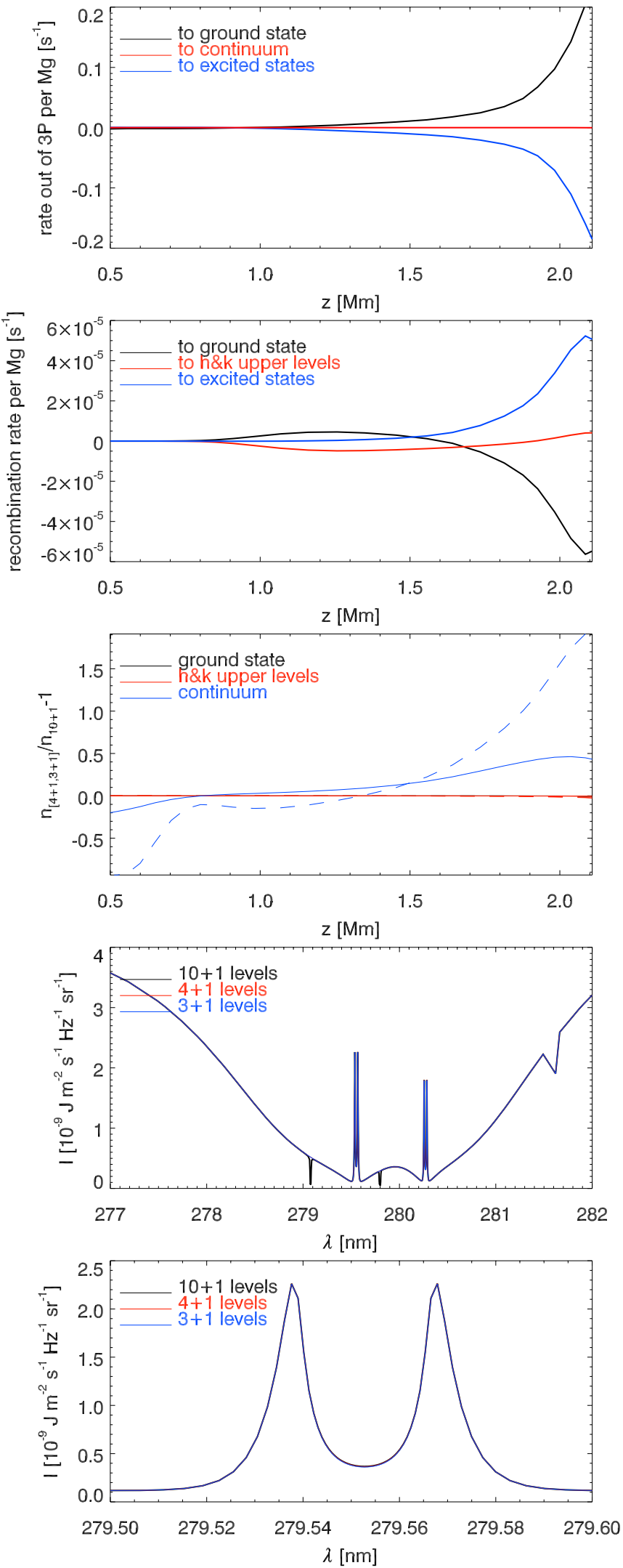

3DMHD
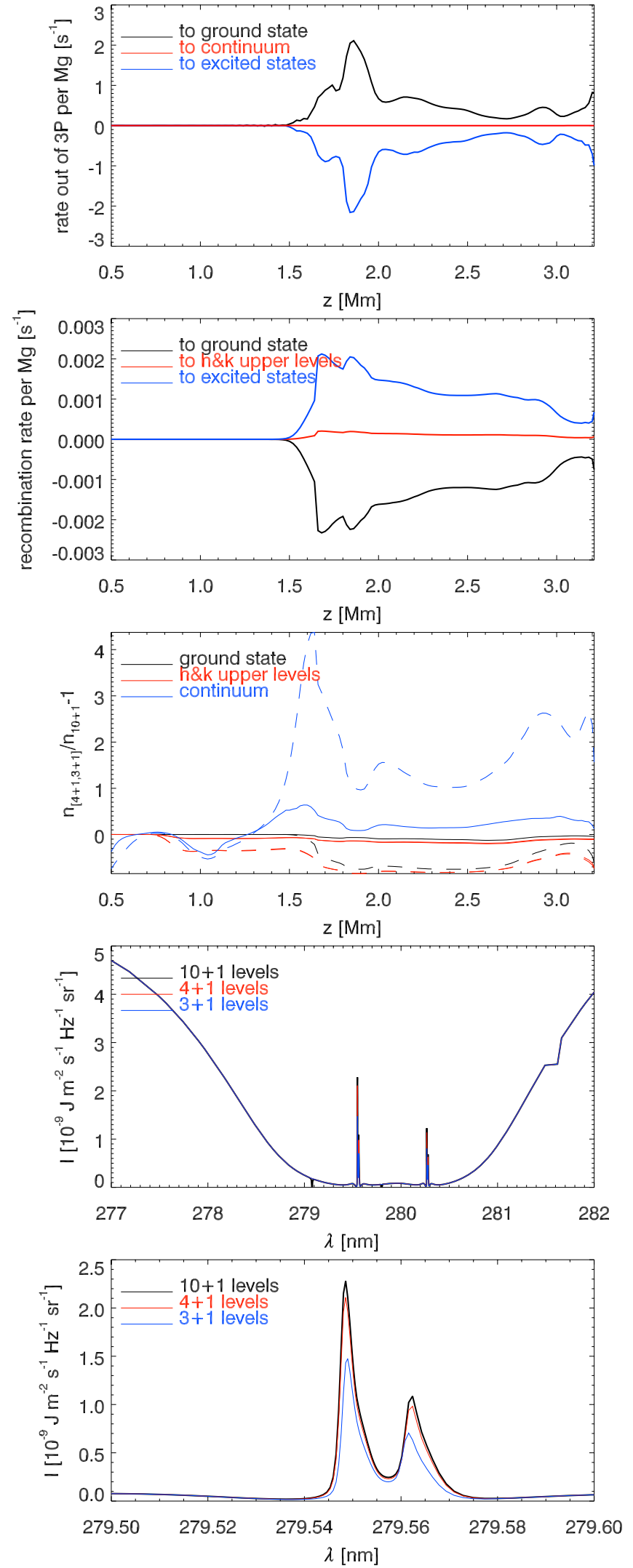

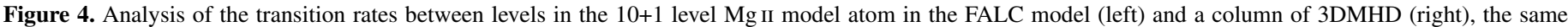

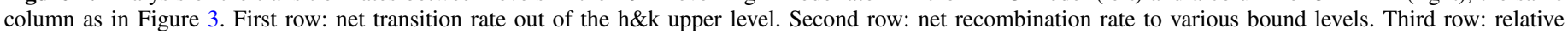

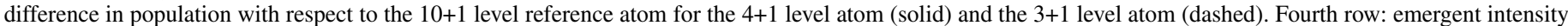
in the h\&k lines for the various model atoms. Fifth row: emergent intensity in the line core of the k line for the various model atoms.

(A color version of this figure is available in the online journal.) 


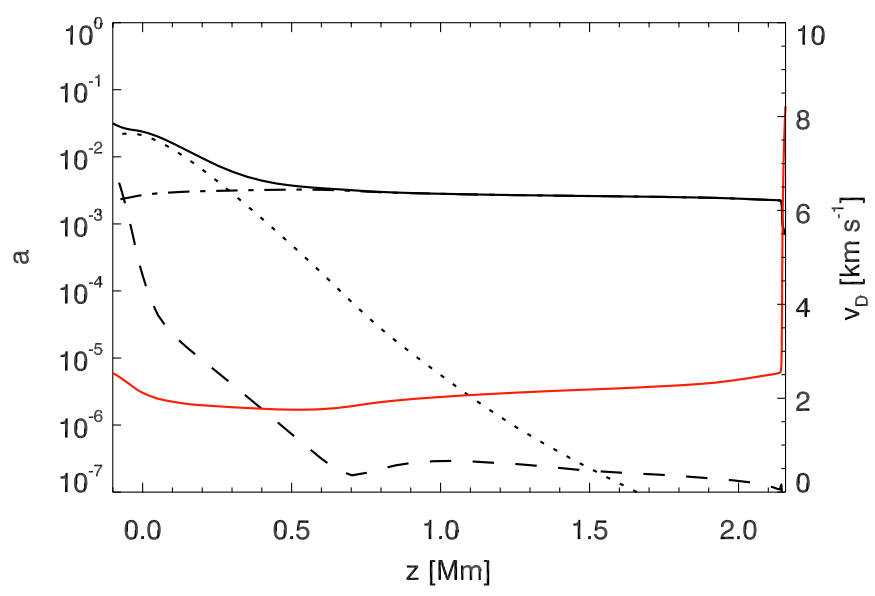

Figure 5. Voigt $a$ damping parameter and the Doppler width $\sqrt{2 k T / m}$ for the $\mathrm{Mg}$ II $\mathrm{k}$ line in the FALC atmosphere. The total damping is shown in solid black (left-hand scale) with the contributions from radiative damping (dash-dotted), Van der Waals broadening (dotted), and the quadratic Stark effect (dashed). The Doppler width is shown in solid red (right-hand scale).

(A color version of this figure is available in the online journal.)

bound states and depopulated to the ground state. The rate to the continuum is negligible.

The second row shows the net recombination rate to the various bound levels. Here the situation is more complex. In FALC below $z=1.5 \mathrm{Mm}$, recombination is to the ground state and ionization is from the h\&k upper levels. Above $1.5 \mathrm{Mm}$, the situation is different, there ionization is from the ground state and recombination is to the higher-lying excited bound states. The same holds for the 3DMHD column.

In conclusion, the $h \& \mathrm{k}$ lines are effectively in detailed balance from the photosphere up to the mid chromosphere. In the upper chromosphere, where the line core forms, h\&k are part of an ionization/recombination loop, where recombination occurs through the highly excited levels, followed by a cascade down through the h\&k lines to the ground state after which ionization occurs again.

We then proceeded to study the differences that occur with smaller model atoms.

We created the simplest model atom capable of reproducing the h\&k lines, which is a three-level plus continuum atom, with the ground state and the two $3 p^{2} P$ states. However, this atom cannot support the ionization/recombination loop described above.

The smallest model atom that can support the loop is a fourlevel plus continuum atom, the same as the $3+1$ level atom, but with an artificial level included that mimics the effect of the excited states above the $3 p^{2} P$ states. This level was created by merging all these excited states so that the total rates in and out of the levels are roughly conserved in the new merged level. Its energy is the average of all the merged levels weighted with their statistical weight.

We then solved the radiative transfer of these $3+1$ and $4+1$ level atoms for the same two models, and plot the relative population difference with the $10+1$ level atom in the third row of Figure 4. In FALC, the h\&k upper and lower level populations are unchanged, but the population of the continuum is very different. As expected, the 4+1 level atom does a better job than the $3+1$ level atom in reproducing the full atomic model populations. In 3DMHD the situation is very different. The $3+1$ level atom has too low h\&k upper and lower level populations, leading to a too low opacity, and a different ratio of the upper and lower level populations, leading to a different source function. The 4+1 level atom does a much better job in reproducing the populations from the full atom.

This is further demonstrated in the fourth row of Figure 4, which shows the emergent intensity for the three different model atoms in the h\&k lines, and the fifth row, which shows the core of the $\mathrm{k}$ line. The line wings are nearly identical, but the $3+1$ level atom fails to reproduce the $10+1$ level atom line core in $3 \mathrm{DMHD}$. In contrast, the $4+1$ level atom does a very good job, with only a slight difference in the $\mathrm{k}_{2 V}$ and $\mathrm{k}_{2 R}$ peak intensities.

We compared the absolute value of the relative difference in emergent intensity between the $3+1$ and 4+1 level atoms and the $10+1$ level atom for a $y z$-slice through the 3DMHD snapshot. The $4+1$ level atom has a maximum average deviation of $3 \%$. Inspection of all individual columns in the slice shows that the difference in any given column is never larger than $11 \%$, whereas the $3+1$ level atom shows differences larger than $50 \%$.

We conclude that it is possible to accurately model the h\&k lines with a $4+1$ level model atom. Further simplification to a $3+1$ level atom leads to significant errors in the line core intensity.

\section{BROADENING IN THE h\&k LINES}

In this section, we discuss the various broadening mechanisms of the $\mathrm{h} \& \mathrm{k}$ lines. We investigate only the $\mathrm{k}$ line, as the $\mathrm{h}$ line behaves nearly identically. Figure 5 shows the various contributions to the damping and the Doppler width of the $\mathrm{k}$ line in the FALC model.

The h\&k lines are resonance lines; the radiative damping is the largest contribution to the total damping in the chromosphere $(z>500 \mathrm{~km})$. Van der Waals broadening is the dominant contribution in the photosphere. Quadratic Stark broadening is only of minor importance. The Voigt $a$ parameter varies from $2.5 \times 10^{-3}$ in the chromosphere to $2.5 \times 10^{-2}$ in the photosphere. The h\&k lines have very broad wings due to their large opacity in the photosphere. In the FALC model, the combined h\&k line opacity at $z=200 \mathrm{~km}$ becomes equal to the continuum opacity at $277.25 \mathrm{~nm}$ and $282.34 \mathrm{~nm}$, more than $2 \mathrm{~nm}$ away from the nearest h\&k line-center frequency. However, the theoretical continuum intensity is never reached in a solar spectrum. The haze of overlapping UV lines prevents the intensity of reaching the "true" continuum (Lemaire \& Samain 1989).

Magnesium has an atomic weight of $24.3 \mathrm{u}$, and a corresponding Doppler width $\sqrt{2 k T / m}$ between 2 and $3 \mathrm{~km} \mathrm{~s}^{-1}$ for typical chromospheric temperatures.

\section{COUPLING BETWEEN h\&k UPPER LEVELS AND THE RELATIVE HEIGHT OF THE EMISSION PEAKS}

Because of the small energy difference between the $\mathrm{h} \& \mathrm{k}$ upper levels, inelastic collisions with neutral hydrogen are important for the transition rates between these levels, in addition to collisions with electrons. In the photosphere and lower chromosphere, the collisions with neutral hydrogen are more important than collisions with electrons due to the lowionization degree of hydrogen there. This is demonstrated in Figure 6, which compares various rate coefficients out of the upper level of the $\mathrm{Mg}$ II $\mathrm{k}$ line in the FALC atmosphere. Collisional de-excitation to the $\mathrm{Mg}$ II $\mathrm{h}$ upper level by neutral hydrogen is dominant between $0 \mathrm{Mm}$ and $1.1 \mathrm{Mm}$ height, above 1.1 Mm de-excitation by electrons dominates. For comparison, we show the collisional de-excitation to the ground state (red), which is comparable to the de-excitation to the $\mathrm{Mg}$ II $\mathrm{h}$ upper 


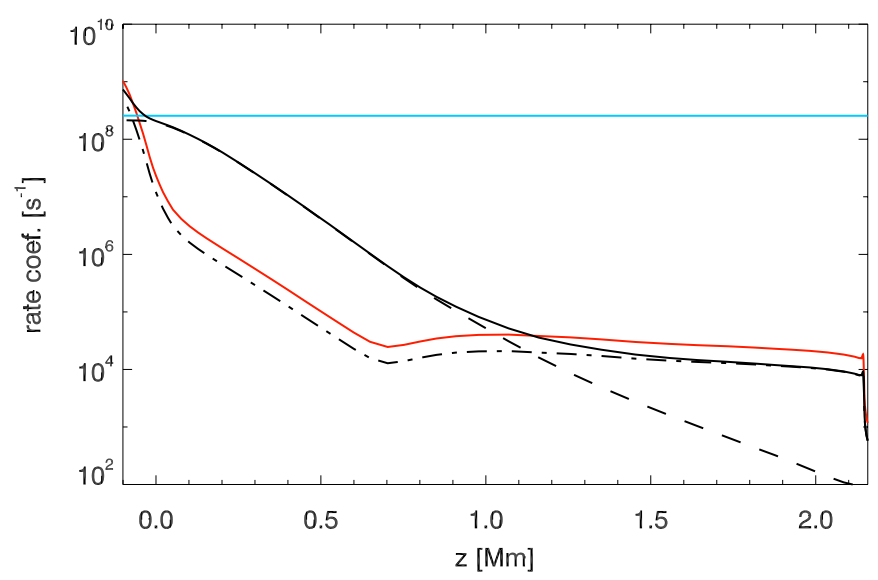

Figure 6. Transition rate coefficients out of the upper level of the $\mathrm{Mg}$ II $\mathrm{k}$ line in the FALC atmosphere. Solid black: total collisional rate coefficient to the upper level of the $\mathrm{Mg}$ II h line, with contribution due to collisions with neutral hydrogen (dashed) and electrons (dot-dashed). For comparison, the radiative rate coefficient to the ground state (blue) and the collisional rate coefficient to the ground state (red) are also shown.

(A color version of this figure is available in the online journal.)

level. In addition, we show the radiative de-excitation to the ground state (blue), which is orders of magnitude larger than the collisional rates, illustrating that $\mathrm{Mg}$ II $\mathrm{k}$ is strongly scattering. The fact that the collisional rates between the levels are much smaller than the spontaneous radiative de-excitation rate to the ground state means that the h\&k upper levels are only weakly coupled with each other and significant differences in the source function can be expected. This is indeed the case, the $\mathrm{k}$ line typically has stronger emission peaks than the $\mathrm{h}$ line in both observations (e.g., Tousey 1967) and theoretical calculations. Interestingly, the observations also show a larger peak height ratio in plage than in the quiet Sun, indicating a sensitivity to the temperature structure in the atmosphere.

The Ca II H\&K lines show a similar effect, which was explained by Linsky (1970). In short, the Ca II K line has twice the opacity as the $\mathrm{H}$ line, and hence its source function thermalizes at a slightly larger height than the source function of the $\mathrm{H}$ line. This means that in a chromosphere with an outwardly increasing temperature, the $\mathrm{K}$ source function follows the temperature rise more strongly than the $\mathrm{H}$ line, and hence the $\mathrm{K}$ emission peaks are higher.

Linsky \& Avrett (1970) argued that the MgII h\&k lines should behave qualitatively the same as Ca II H\&K. We confirm this by analyzing the formation difference between $h \& \mathrm{k}$ in the FALC atmosphere in Figure 7. The upper left panel shows the h\&k line profiles, with a higher emission-peak intensity for the $\mathrm{k}$ line. The upper right panel shows the ratio of the non-LTE upper-level populations, indicating that the $\mathrm{k}$ line has a higher source function $S$ because (neglecting PRD effects) $S_{k} / S_{h} \approx(1 / 2) n_{k} / n_{h}$ as the lines share a common lower level and the ratio of the statistical weights of the upper levels is 2 (Rutten 2003, Section 2.6.2). The near equality of the population ratio with the ratio of the radiative rates in the h\&k lines shows that the populations are set completely by the line radiation. The lower panels finally show a comparison of the source function and height of optical depth unity. Because the k opacity is twice as high as the $h$ opacity, optical depth unity in the emission peaks in the $\mathrm{k}$ line is reached already at larger heights, and the source function is larger (lower left panel). This leads to the larger emission-peak height. The k-line source function is also higher around optical depth unity at line center, leading to a larger line-center intensity.

\section{EFFECT OF OPTICAL PUMPING BY H I Ly $\beta$}

The $\mathrm{Mg}$ II atom has two radiative transitions between the $5 p^{2} P$ states and the $3 s^{2} S$ ground state, at wavelengths close to $102.6 \mathrm{~nm}$, which is in the red wing of the $\mathrm{H}_{\mathrm{I}} \mathrm{Ly} \beta$ line. Optical pumping in these transitions from the ground state through the large radiation field caused by the Lyman line could influence the formation of the h\&k lines by creating a pathway for population of the h\&k upper levels.

We investigated this effect by computing the simultaneous non-LTE solution of a five-level plus continuum $\mathrm{H}$ I and a 21 level plus continuum $\mathrm{Mg}$ II atom with and without these two transitions in the FALC atmosphere and one column from the 3DMHD snapshot. In both cases, neglecting the $3 s^{2} S-5 p^{2} P$ transitions has a negligible effect on the h\&k lines.

\section{IMPORTANCE OF 3D RADIATIVE TRANSFER}

The Mg II h\&k lines are strongly scattering as illustrated by Figure 6, which shows that the probability for radiative deexcitation from the upper levels is four orders of magnitude larger than the probability for collisional de-excitation. This means that the source function is largely set by the radiation field, which in turn depends on the atmospheric structure. If the typical effective photon mean free path is comparable to or larger than the scale of the horizontal inhomogeneities in the atmosphere, then effects of horizontal radiation transport are important. Typically, this leads to a smoothing of the radiation field and a corresponding smoothing of the source function.

We estimate the $3 \mathrm{D}$ effects by using the expression for an infinitely sharp-lined, two-level atom with isotropic scattering. In that case the effective photon mean free path can be expressed in terms of the opacity $\alpha$ and the photon destruction probability $\epsilon$ :

$$
l^{\star}=\frac{1}{\alpha_{\nu} \sqrt{\epsilon}} .
$$

The photon destruction probability is given by

$$
\epsilon=\frac{C_{\mathrm{ul}}}{C_{\mathrm{ul}}+A_{\mathrm{ul}}+B_{\mathrm{ul}} B_{v}},
$$

with $C_{\mathrm{ul}}$ being the coefficient for collisional de-excitation, $A_{\mathrm{ul}}$ and $B_{\mathrm{ul}}$ being the Einstein coefficients for spontaneous deexcitation and induced de-excitation, respectively, and $B_{v}$ being the Planck function.

We computed $l^{\star}$ for the Mg II $\mathrm{k}$ line in the 3DMHD atmosphere, from a 3D non-LTE CRD computation with Multi3d taking as opacity the line-center absorption coefficient for an outgoing vertical ray. In Figure 8, we compare it with the average height of formation of the $\mathrm{k}_{1}, \mathrm{k}_{2 R}, \mathrm{k}_{2 \mathrm{~V}}$, and $\mathrm{k}_{3}$ spectral features. Based on this rough estimate, we expect that the $\mathrm{k}_{1}$ and $\mathrm{k}_{2 R}$ intensities are not affected by 3D effects. The $\mathrm{k}_{2 V}$ peak is marginally affected at the horizontal resolution of the radiative transfer computation $(96 \mathrm{~km}$, compared to a median photon mean free path of $\approx 300 \mathrm{~km}$ ), while $\mathrm{k}_{3}$ is strongly affected.

We confirm this in Figure 9, where we compare the vertically emergent intensity in $\mathrm{k}_{2 V}, \mathrm{k}_{3}$, and $\mathrm{k}_{2 R}$ computed in $3 \mathrm{D}$ and $1 \mathrm{D}$, but assuming CRD. For each pixel in the image, we determined the wavelength of the spectral features using an automated algorithm. Because of the very complex, multiplepeaked profiles, this algorithm does not correctly identify the spectral locations of the features for all pixels. These pixel 

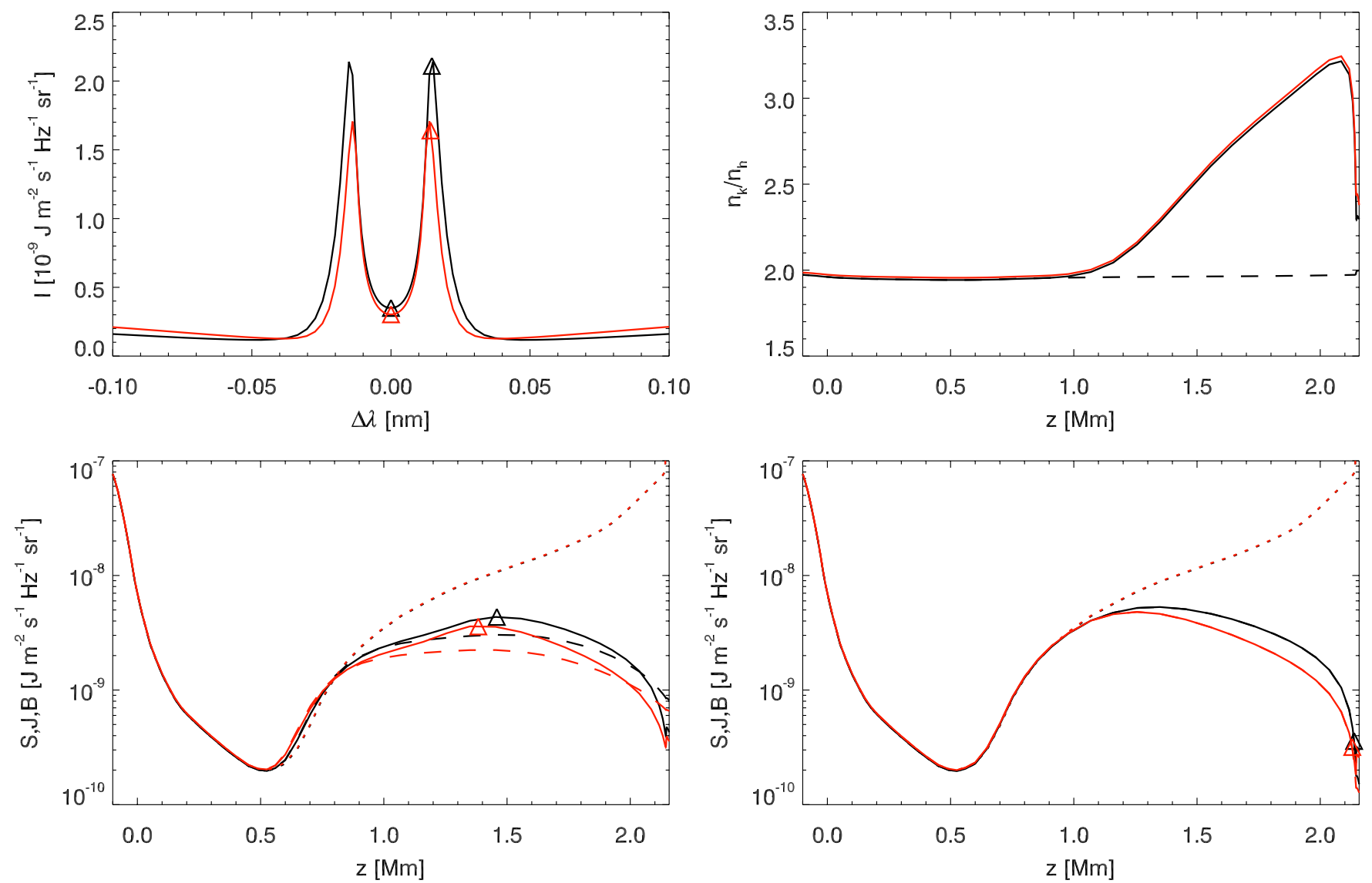

Figure 7. Difference in the emission-peak height between the $h \& k$ lines in the FALC model atmosphere. The triangles indicate wavelengths analyzed in the lower panels. Upper left: $\mathrm{h}$ (red) and $\mathrm{k}$ (black) line core profiles as function of wavelength shift away from line center. Upper right: the ratio of the $\mathrm{h} \& \mathrm{k}$ upper level populations $n_{\mathrm{k}} / n_{\mathrm{h}}$ in non-LTE (solid black) and LTE (dashed black). The ratio of the upward radiative rates $R_{\mathrm{k}} / R_{\mathrm{h}}$ is overplotted in red. Lower left: source function (solid), angle-averaged radiation field (dashed), and Planck function (dotted) at the red emission peak (indicated by the triangles in the upper left panel) for the k line (black) and the $\mathrm{h}$ line (red). The triangles indicate optical depth unity at this wavelength. Lower right: same as lower left, but now for line center.

(A color version of this figure is available in the online journal.)

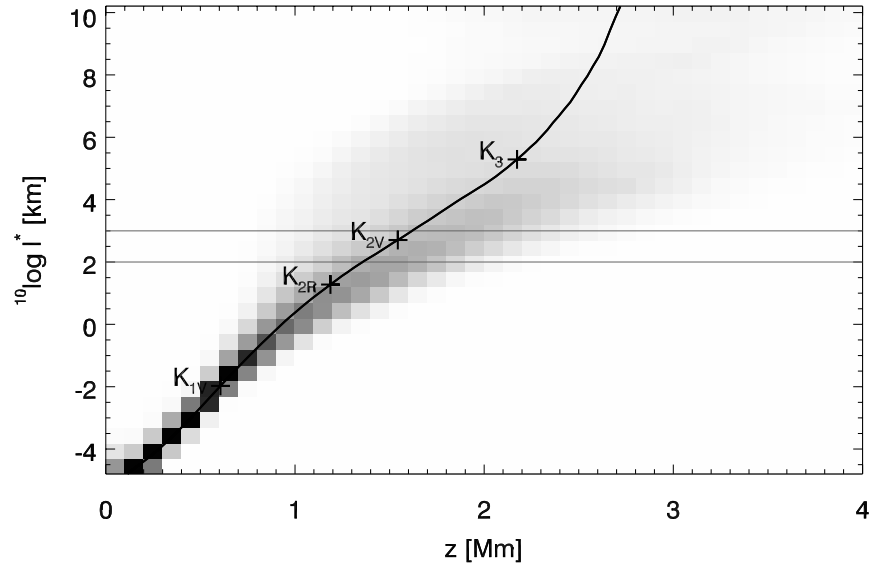

Figure 8. Distribution of photon mean free paths as a function of height in the 3DMHD model atmosphere at the Mg II $\mathrm{k}$ line center. The median of the mean free path as a function of height is shown in solid black. The crosses indicate the average $\tau=1$ heights at wavelengths for various spectral features in the average profile: the minimum on the blue side of the central emission peaks $\left(\mathrm{k}_{1 \mathrm{~V}}\right)$, the red $\left(\mathrm{k}_{2 \mathrm{R}}\right)$ and blue $\left(\mathrm{k}_{2 \mathrm{~V}}\right)$ emission peaks, and the line-center depression $\left(\mathrm{k}_{3}\right)$. The gray lines indicate free paths of $100 \mathrm{~km}$ and $1000 \mathrm{~km}$ to guide the eye.

locations show up as noise (dark pixels in $\mathrm{k}_{2 V}$ and $\mathrm{k}_{2 R}$, bright pixels in $\mathrm{k}_{3}$ ).

The $\mathrm{k}_{2 V}$ and $\mathrm{k}_{2 R}$ spectral features are weakly affected by $3 \mathrm{D}$ effects. Typically, the dark areas are brighter in three dimensions than in one dimension, and the 3D image is slightly fuzzier. The $\mathrm{k}_{3}$ image is strongly affected by $3 \mathrm{D}$ effects. The 3D image is much fuzzier, and there is a much more pronounced fibril structure than in one dimension. In one dimension, there are only fibrils visible in the central region, where the chromospheric magnetic field is strongest. Elsewhere, the 1D image shows shock waves with a structure very similar to what is seen in the $\mathrm{k}_{2 V}$ image.

The same information is shown as the joint probability density function (JPDF) of the 3D and 1D intensities in Figure 10. It confirms the impression from the images in Figure 9: 3D effects are strong for $\mathrm{k}_{3}$, and much weaker for $\mathrm{k}_{2 V}$ and $\mathrm{k}_{2 R}$.

For the two emission peaks, the largest $3 \mathrm{D}$ effects occur at low intensities, where the 3D intensity is larger than the 1D intensity. This is caused by the sideways illumination of cool parts of the atmosphere by hotter neighboring locations. This illumination leads to a higher angle-averaged radiation field in $3 \mathrm{D}$, and thus to a higher source function and emergent intensity. The reverse happens for locations with high $\mathrm{k}_{2 V}$ and $\mathrm{k}_{2 R}$ intensities; there the $3 \mathrm{D}$ radiation field is lower than in $1 \mathrm{D}$ owing to the lateral averaging. The $\mathrm{k}_{2 R}$ peak intensity is slightly less influenced by $3 \mathrm{D}$ effects than $\mathrm{k}_{2 V}$, in agreement with the lower formation height of $\mathrm{k}_{2 R}$ (see Figure 8).

The $\mathrm{k}_{3}$ intensity minimum shows only a weak correlation between 1D and 3D intensities, as expected based on the different appearance of the 1D and 3D images in Figure 9.

We performed the same analysis for the $\mathrm{h}$ line and reached the same conclusions. 


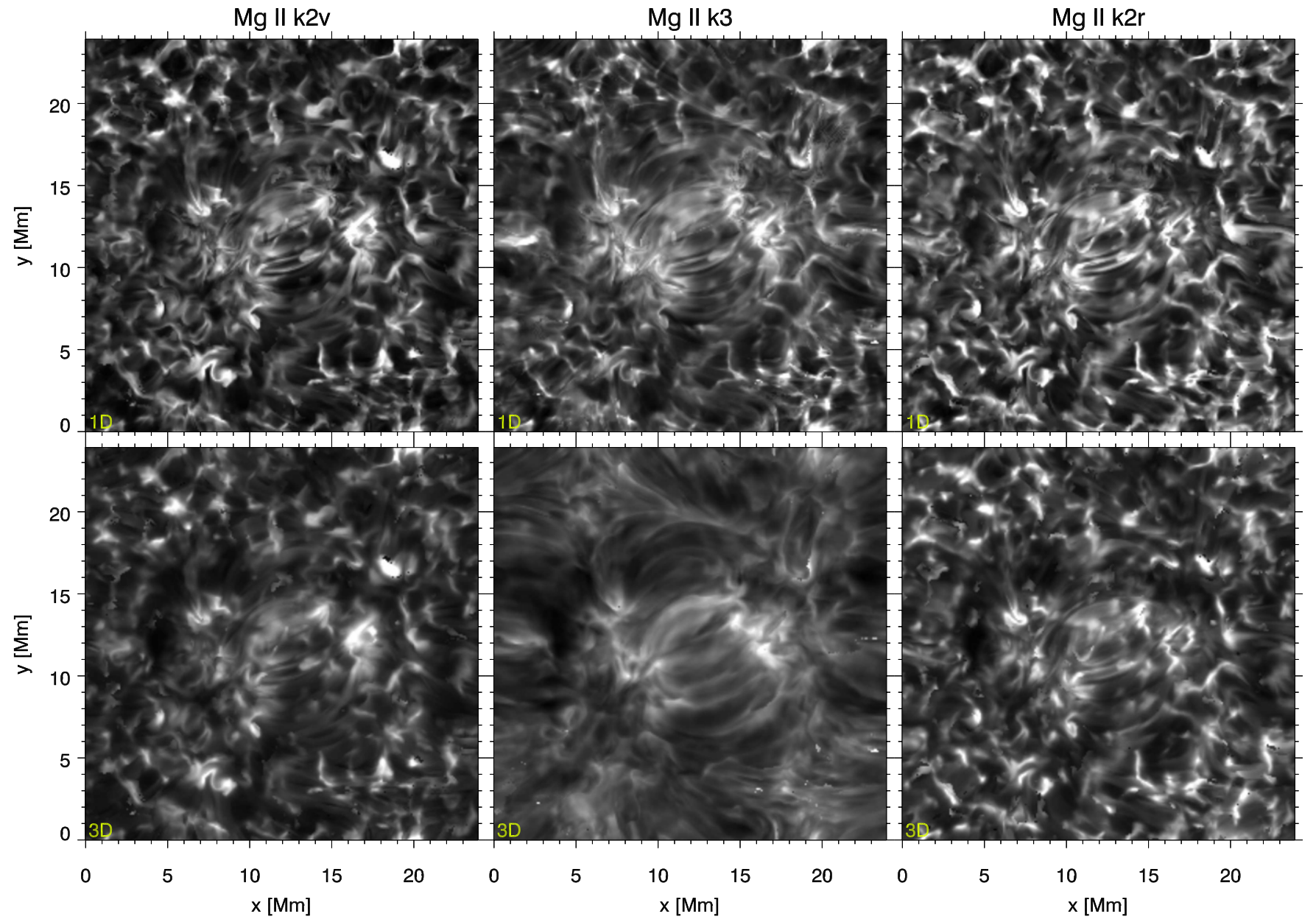

Figure 9. Comparison of the vertically emergent intensity in the $\mathrm{Mg}$ II $\mathrm{k}$ line core between $1 \mathrm{D}$ (top row) and 3D (bottom row) computations, performed assuming CRD. The columns show the intensity for various spectral features indicated above the top panels. The top and bottom panels in each column have the same brightness scale so the 1D and 3D intensity can be compared directly.

(A color version of this figure is available in the online journal.)
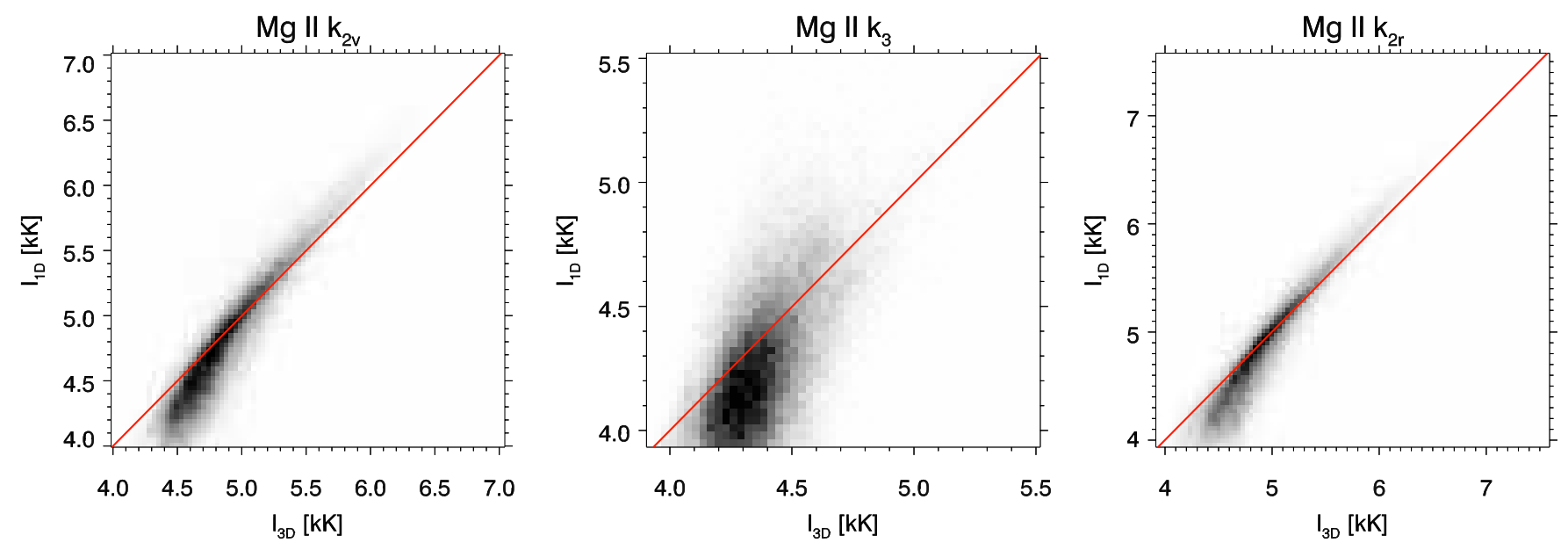

Figure 10. Joint probability density functions of the vertically emergent intensity computed in 3D and in $1 \mathrm{D}$. Left: $\mathrm{k}_{2 V}$; middle: $\mathrm{k}_{3}$; right: $\mathrm{k}_{2 R}$. The red line is the line $I_{1 \mathrm{D}}=I_{3 \mathrm{D}}$

(A color version of this figure is available in the online journal.)

\section{EFFECT OF PARTIAL REDISTRIBUTION}

We now turn our attention to the effect of PRD on the emergent line profiles. The $\mathrm{Mg}$ II $\mathrm{h} \& \mathrm{k}$ lines are strongly affected by the effect of PRD. Owing to the low particle density in the chromosphere, the average time between collisions is large compared to the lifetime of an $\mathrm{Mg}$ II ion in the upper levels of the lines. Therefore, the frequency of the absorbed and emitted photon in a scattering process are correlated and the assumption of CRD is invalid. We demonstrate this effect in Figure 11, which displays a comparison of vertically emergent line profiles computed with $R H$ in PRD and CRD for two different columns 


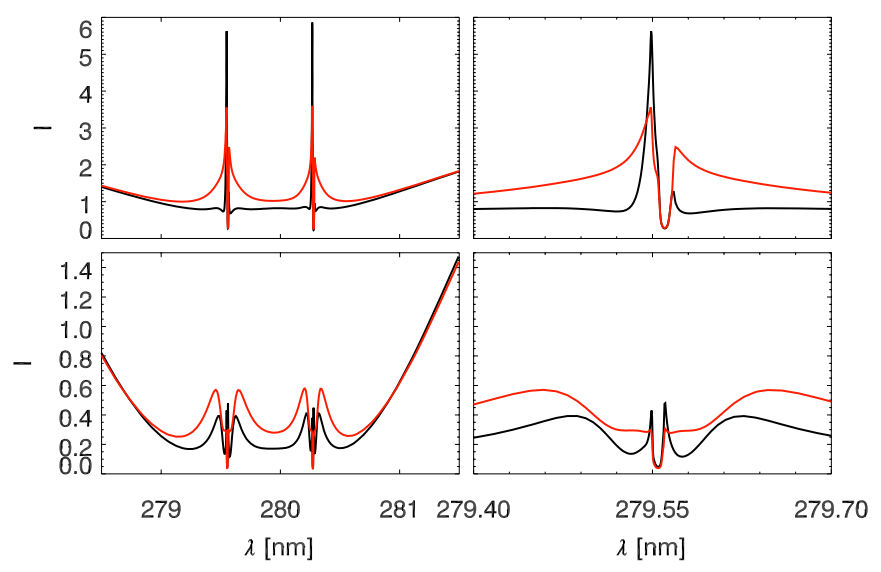

Figure 11. Comparison of the emergent $\mathrm{Mg}$ II h\&k line profiles in two locations of the 3DMHD atmosphere (top and bottom rows). The left-hand column shows both h\&k lines, the right-hand column shows a zoom of the line core of the $\mathrm{k}$ line. The black curve is the PRD profile and the red curve is the CRD profile. The intensity is given in units of $10^{-9} \mathrm{~J} \mathrm{~m}^{-2} \mathrm{~s}^{-1} \mathrm{~Hz}^{-1} \mathrm{sr}^{-1}$.

(A color version of this figure is available in the online journal.)

in the 3DMHD atmosphere. These computations were done assuming the 1D plane-parallel approximation.

Inclusion of PRD has the strongest effect in the inner wing of the line profile. Typically, the PRD computations yield a lower inner-wing intensity (around the $\mathrm{k}_{1}$ minima) and a higher intensity in the emission peaks $\left(\mathrm{k}_{2 V}\right.$ and $\left.\mathrm{k}_{2 R}\right)$. The intensity in the $\mathrm{k}_{3}$ minimum is only weakly influenced by PRD effects. Images of the $\mathrm{k}_{3}$ intensity computed in PRD and CRD from the 3DMHD atmosphere appear nearly identical as shown in Figure 12.

A more quantitative comparison is given in Figure 13, which shows the JPDF of the intensity in $\mathrm{Mg}$ II $\mathrm{k}_{3}$ computed in PRD and CRD. At low intensities there is a very tight correlation between the PRD and CRD computations, at higher intensities the correlation becomes somewhat weaker and the PRD intensity tends to be higher than the CRD intensity. In general, assuming CRD for the $\mathrm{k}_{3}$ minimum is a very good approximation.

\section{DISCUSSION AND CONCLUSIONS}

We studied the basic formation properties of the $\mathrm{Mg}$ II $\mathrm{h} \& \mathrm{k}$ lines in models of the solar atmosphere.

We first investigated the time-dependent, non-equilibrium ionization balance of $\mathrm{Mg}$ II $/ \mathrm{Mg}$ III and found that whenever the temperature is high enough to give a significant fraction of $\mathrm{Mg}$ III, the relaxation time is short. We therefore conclude that statistical equilibrium is a good approximation for the formation of the $\mathrm{Mg}$ II h\&k lines.

We then investigated the influence of $\mathrm{Mg}$ I on the line formation and found that treating neutral magnesium in LTE as a source of background opacity leads to lower intensity in the outer line wings. The h\&k line cores are unaffected. If one is interested in the outer wing intensities, one should include $\mathrm{Mg}$ I in the non-LTE model atom. We used a model with 65 bound levels of $\mathrm{Mg}$ I. It is possible that a smaller number of $\mathrm{Mg}$ I levels is sufficient to model the effect on the h\&k lines. If so, this would reduce the required computational effort significantly.

We then constructed a minimal model atom that models the $\mathrm{h} \& \mathrm{k}$ lines. This model atom contains four bound levels and a continuum. The bound levels are the $\mathrm{Mg}$ II ground state, the $2 p^{6} 3 p$ doublet which are the upper levels of the lines, and a higher-lying artificial level that is required to correctly recover the $\mathrm{Mg}$ II-Mg III ionization balance. This artificial level acts as a target level for the recombination of $\mathrm{Mg}$ III. Omitting this level leads to an incorrect ionization balance in the upper chromosphere and too low emission peaks.

Our investigation of the difference between the $h$ line and the $\mathrm{k}$ line confirms the conjecture by Linsky \& Avrett (1970) that the stronger emission in the $\mathrm{k}$ line is because the $\mathrm{k}$ line has twice the opacity of the $h$ line. This larger opacity causes a larger formation height and a stronger coupling of the source function to the gas temperature at the locations in the atmosphere where the emission peaks form.

The Mg II h\&k lines are scattering lines, and the emergent intensity is influenced by 3D effects in the line core. The intensity in the emission peaks is only mildly affected: in 3D contrast is decreased compared to $1 \mathrm{D}$ computations, but the overall appearance of the images computed from the 3DMHD model remains the same. The $\mathrm{k}_{3}$ and $\mathrm{h}_{3}$ intensity minima are strongly affected by 3D effects: 3D images are fuzzier and show long extended fibrils. Intensity minimum images computed in 1D show only a very weak imprint of fibrils and instead exhibit a shock wave pattern similar to images of the intensity in the emission peaks.

The assumption of CRD is not valid for $\mathrm{Mg}$ II h\&k. The line profiles must be computed assuming PRD, otherwise the linewing intensity up to and including the emission peaks will be wrong. However, the intensity in the $\mathrm{k}_{3}$ and $\mathrm{h}_{3}$ minima computed in CRD is very similar to the correct intensity computed in PRD.

Based on this analysis we can now answer the question how best to model the Mg II h\&k lines in time series of snapshots from large 3D RMHD models.

The current state of the art in unpolarized non-LTE radiative transfer modeling allows for quick computation of line profiles including PRD treating each column in the snapshot as an independent 1D plane-parallel atmosphere. Radiative transfer computations taking the full 3D structure into account are also possible, but only assuming CRD. Non-LTE computations including both PRD and 3D simultaneously are currently not possible: inclusion of PRD in the non-LTE computations occasionally leads to instabilities in the Lambda-iteration. In $1 \mathrm{D}$, this would only lead to a non-converged column, but in 3D the whole computation would fail. This is a problem for modeling the h\&k lines, which are influenced both by $3 \mathrm{D}$ and PRD effects. Our results show, however, that by combining 1D PRD and 3D CRD computations one can capture the essentials of the true profile. The profile up to and including the emission peaks is well approximated by 1D PRD. The central depression can be modeled reasonably well with 3D CRD computations. We stress that a 1D CRD approach is not realistic and should not be used.

While this combined approach is not truly satisfactory, it nevertheless opens up the possibility of modeling the $\mathrm{Mg}$ II $\mathrm{h} \& \mathrm{k}$ lines with enough realism to study their formation in $3 \mathrm{D}$ numerical models of the solar atmosphere and determines their potential as atmospheric diagnostics. We report on the results of such a study in Paper II of this series.

J.L. recognizes support from the Netherlands Organization for Scientific Research (NWO). This research was supported by the Research Council of Norway through the grant "Solar Atmospheric Modeling," from the European Research Council under the European Union's Seventh Framework Programme (FP7/2007-2013)/ERC grant agreement No. 291058, and through grants of computing time from the Programme for 


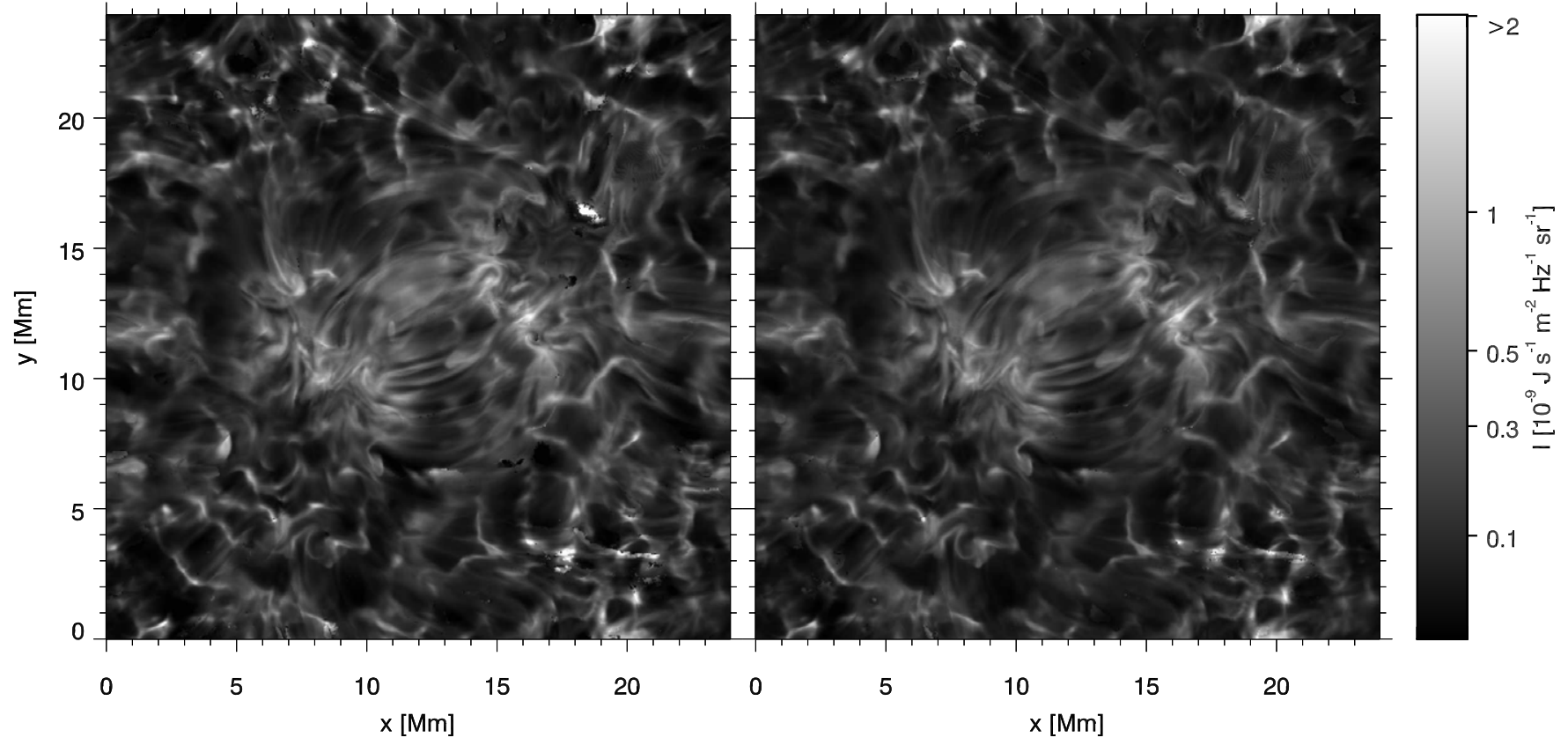

Figure 12. Comparison of the $\mathrm{k}_{3}$ intensity computed in 1D with PRD (left-hand panel) and CRD (right-hand panel). Both images have the same brightness scale, given on the right. Columns where the wavelength of $\mathrm{k}_{3}$ is misidentified appear as white pixels.

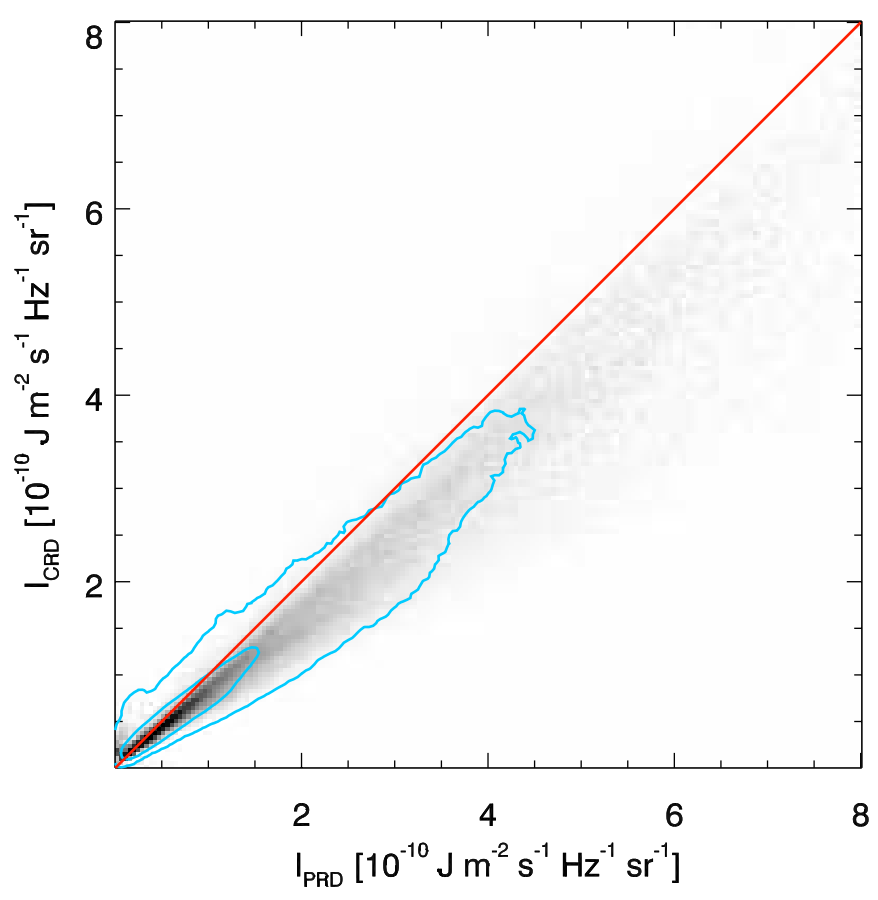

Figure 13. Joint probability density function of the intensity in $\mathrm{Mg}_{\mathrm{II}} \mathrm{k}_{3}$ computed in PRD (horizontal axis) and CRD (vertical axis). Darker color indicates a higher number of pixels in the bin. The image has been clipped and scaled to bring out the distribution at high intensities. The red line indicates $I_{\mathrm{PRD}}=I_{\mathrm{CRD}}$. The inner blue contour encloses $50 \%$ of all pixels, the outer blue contour encloses $90 \%$ of all pixels.

(A color version of this figure is available in the online journal.)

Supercomputing of the Research Council of Norway and computing project s1061 from the High End Computing Division of NASA. T.M.D.P. was supported by the NASA Postdoctoral Program at Ames Research Center (grant NNH06CC03B). B.D.P. acknowledges support from NASA grants NNX08AH45G, NNX08BA99G, NNX11AN98G, and NNG09FA40C (IRIS).
We thank Paul Barklem for pointing out the paper on collisions with neutral hydrogen.

\section{REFERENCES}

Abia, C., \& Mashonkina, L. 2004, MNRAS, 350, 1127

Allen, M. S., \& McAllister, H. C. 1978, SoPh, 60, 251

Anstee, S. D., \& O'Mara, B. J. 1995, MNRAS, 276, 859

Arnaud, M., \& Rothenflug, R. 1985, A\&AS, 60, 425

Artzner, G., Vial, J. C., Lemaire, P., Gouttebroze, P., \& Leibacher, J. 1978, ApJL, 224, L83

Asplund, M., Grevesse, N., Sauval, A. J., \& Scott, P. 2009, ARA\&A, 47, 48

Barklem, P. S., \& O’Mara, B. J. 1998, MNRAS, 300, 863

Bonnet, R. M., Lemaire, P., Vial, J. C., et al. 1978, ApJ, 221, 1032

Burgess, A., \& Chidichimo, M. C. 1983, MNRAS, 203, 1269

Carlsson, M., \& Leenaarts, J. 2012, A\&A, 539, A39

Carlsson, M., Rutten, R. J., \& Shchukina, N. G. 1992, A\&A, 253, 567

Carlsson, M., \& Stein, R. F. 1992, ApJL, 397, L59

Carlsson, M., \& Stein, R. F. 1997, ApJ, 481, 500

Carlsson, M., \& Stein, R. F. 2002, ApJ, 572, 626

Chang, E. S., Avrett, E. H., Noyes, R. W., Loeser, R., \& Mauas, P. J. 1991, ApJL, 379, L79

Cunto, W., Mendoza, C., Ochsenbein, F., \& Zeippen, C. J. 1993, A\&A, 275, L5

Dorfi, E. A., \& Drury, L. O. 1987, JCoPh, 69, 175

Fontenla, J. M., Avrett, E. H., \& Loeser, R. 1991, ApJ, 377, 712

Fontenla, J. M., Avrett, E. H., \& Loeser, R. 1993, ApJ, 406, 319

Gouttebroze, P. 1989, ApJ, 337, 536

Gudiksen, B. V., Carlsson, M., Hansteen, V. H., et al. 2011, A\&A, 531, A154

Hayek, W., Asplund, M., Carlsson, M., et al. 2010, A\&A, 517, A49

Kneer, F., Mattig, W., Scharmer, G., et al. 1981, SoPh, 69, 289

Kohl, J. L., \& Parkinson, W. H. 1976, ApJ, 205, 599

Leenaarts, J., \& Carlsson, M. 2009, in ASP Conf. Ser. 415, The Second Hinode Science Meeting: Beyond Discovery-Toward Understanding, ed. B. Lites, M. Cheung, T. Magara, J. Mariska, \& K. Reeves (San Francisco, CA: ASP), 87

Leenaarts, J., Carlsson, M., Hansteen, V., \& Gudiksen, B. V. 2011, A\&A, 530, A124

Leenaarts, J., Carlsson, M., Hansteen, V., \& Rutten, R. J. 2007, A\&A, 473, 625 Leenaarts, J., Carlsson, M., \& Rouppe van der Voort, L. 2012a, ApJ, 749, 136

Leenaarts, J., Pereira, T., \& Uitenbroek, H. 2012b, A\&A, 543, A109

Lemaire, P., \& Samain, D. 1989, in High Spatial Resolution Solar Observations (Sunspot, MN: National Solar Observatory), 551

Linsky, J. L. 1970, SoPh, 11, 355 
Linsky, J. L., \& Avrett, E. H. 1970, PASP, 82, 169

Mihalas, D. 1978, Stellar Atmospheres (2nd ed.; San Francisco, CA: Freeman), 650

Milkey, R. W., \& Mihalas, D. 1974, ApJ, 192, 769

Monteiro, T. S., Danby, G., Cooper, I. L., Dickinson, A. S., \& Lewis, E. L. 1988, JPhB, 21, 4165

Morrill, J. S., Dere, K. P., \& Korendyke, C. M. 2001, ApJ, 557, 854

Morrill, J. S., \& Korendyke, C. M. 2008, ApJ, 687, 646

Nordlund, A. 1982, A\&A, 107, 1

Ralchenko, Y., Kramida, A., Reader, J., \& NIST ASD Team. 2011, http:// physics.nist.gov/asd3

Rezaei, R., Bruls, J. H. M. J., Schmidt, W., et al. 2008, A\&A, 484, 503

Rutten, R. J. 2003, Radiative Transfer in Stellar Atmospheres, Utrecht Univ. Lecture Notes, 8th ed.

Shull, J. M., \& van Steenberg, M. 1982, ApJS, 49, 351
Sigut, T. A. A., \& Pradhan, A. K. 1995, JPhB, 28, 4879

Skartlien, R. 2000, ApJ, 536, 465

Staath, E., \& Lemaire, P. 1995, A\&A, 295, 517

Tousey, R. 1967, ApJ, 149, 239

Uitenbroek, H. 1997, SoPh, 172, 109

Uitenbroek, H. 2001, ApJ, 557, 389

Uitenbroek, H., \& Briand, C. 1995, ApJ, 447, 453

Vial, J.-C. 1984, LAstr, 98, 211

Vial, J. C., Gouttebroze, P., Artzner, G., \& Lemaire, P. 1979, SoPh, 61,39

Vial, J. C., Salm-Platzer, J., \& Martres, M. J. 1981, SoPh, 70, 325

West, E., Cirtain, J., Kobayashi, K., et al. 2011, Proc. SPIE, 8160, 816010

Woodgate, B. E., Brandt, J. C., Kalet, M. W., et al. 1980, SoPh, 65, 73

Zhao, G., Butler, K., \& Gehren, T. 1998, A\&A, 333, 219 